\title{
Measurement and analysis of resistivity of graphite
}

\author{
Wengang Zheng ${ }^{1, a}$ Zhiyi Zhang $^{2, b}$
}

${ }^{1}$ College of Mechanica Engineering, Beihua University, Jilin Province, People's Republic of China

${ }^{2}$ Engineering Training Center, Beihua University, Jilin Province, People's Republic of China

aemail:985304275@qq.com, ${ }^{\mathrm{b}}$ email:zczhang6@163.com

Keywords: Graphite electrode, Resistivity measurement, Resistivity analysis

\begin{abstract}
The resistivity of graphite electrode is not only an important parameter to reflect the physical characteristics, but also the main technical index of the graphite electrode. In this paper, The independent design and development of the equipment used for measuring resistivity of graphite electrode body of experimental measurements and data analysis, and analysis of the distribution of the bulk resistivity under different electrode plate through change measurement of graphite electrodes at two ends of the main body of the electrode plate shape; at the same time, choose the appropriate location in the different phase of resistivity variation analysis[1] [2].
\end{abstract}

\section{The Principle and Method of Measurement of Resistivity of Graphite Electrode}

The resistivity is also called coefficient of resistance or resistance, it is a conductor by the nature of resistance by a current.Its size is conductor (at room temperature $20^{\circ} \mathrm{C}$ )of resistancemultiplied by the cross-sectional area, With $\rho$ represent, The formula is as follows:

$$
\rho=\frac{U S}{I L}
$$

The measuring method of resistivity of graphite electrode is double bridge method, micro ohm meter method, non-contact measurement method, DC probe pressure drop method and so on[3].DC probe pressure drop method is the international standard method, and it is also a common method in the world, It can be applied to the determination of the resistivity of carbon materials in a large section. This study analyzed by measuring the resistivity of graphite electrodes to provide a theoretical basis for the industrial production of product quality testing, Therefore, the methods used to measure the DC voltage drop method probe. Below is the simple diagram of the actual measurement:

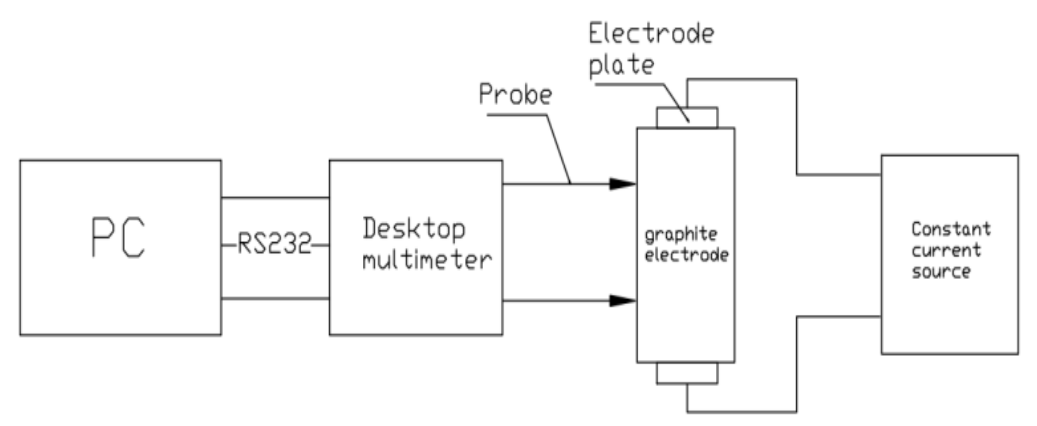

Figure 1 Simple chart of resistivity measurement

Specifications of graphite electrode experiments we used for $\Phi 160 \mathrm{~mm} \times 223 \mathrm{~mm}$,and after the car outside circle processing. During the experiment on both ends of the graphite electrode through the constant current, changing the distance between the probe and the electrode plate in both rectangular and circular electrode plate electrode plate opposite the probe turned condition of phase angle.The upper and lower distance of the graphite electrode is divided into 13 parts, the whole circle is divided into 16 phases. Then the voltage drop between the two probes is measured by using a table top, and the electrical resistivity of the graphite electrode is calculated according to the formula (1). 


\section{Resistivity Measuring Device}

Graphite electrode resistivity measurement of the mechanical requirements: good repeatability and high precision; simple and convenient method of measuring, high level of automation; mechanical transmission smoothly without rapid impact phenomenon; electrode clamping fully; chuck should have sufficient pressure, etc.For other parts, there are some requirements , such as:You must ensure constant output current source; multimeter measurement accuracy must be high enough; the data acquisition is fast enough ${ }^{[5-9]}$, etc.In order to meet the above experimental conditions, the laboratory independently designed and developed a new type of measuring equipment, as shown in figure 2:

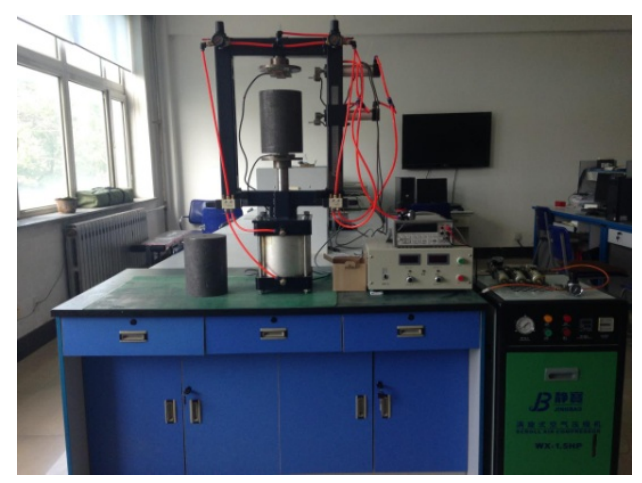

Figure 2 New type of graphite electrode resistivity measurement equipment

\section{The choice of testing equipment.}

The current provided for the graphite electrode plate must be constant and can be easily adjusted.DlC-6000C-30-50 type power supply can meet the output constant current and its resolution can reach 0.01A.Desktop million table is using the Agilent 34401A digital multimeter, it has resolution, 24 hours $0.0015 \%$ basic DC voltage accuracy, accurate and can be repeated to obtain a numerical test.

\section{The motion control system of measuring device.}

The power source part of this device is provided by an air pump,In order to realize the measurement of the process automation we use SIEMENS PLC S7-200 control air solenoid valve to achieve the clamping of the graphite electrode, and the extension of the probe and the return.

\section{Data Acquisition and Date Analysis}

Desktop digital multimeter between two probe real-time measurement of voltage drop and the size of unit level and numerical changes rapidly, artificial reading will cause large errors, therefore we use the computer automatically, the interval of the voltage value into the collection. Thus it can reduce the people for the burden of the operation but also to ensure the accuracy of the measurements.

In addition to the size of the graphite electrode and other factors, the electric resistivity of the graphite electrode can be affected by the measurement of both ends of the electrode plate change.Therefore the experimental design of the two different disk electrode: rectangular electrode plate, a round disk electrode according to practical industrial requirements. We also study the in rectangular electrode plate phase on the resistivity of graphite electrode effects.

The current density of different electrodes is different, but the influence of different current density on the resistivity of graphite electrode is very large,So, how to choose the appropriate disk electrode resistivity of graphite electrode measurement is very important. Rectangular disk electrode size width $35 \mathrm{~mm}$, long $165 \mathrm{~mm}$, 40mm thick, round disk electrode size $100 \mathrm{~mm}$, 40mm thick, production of materials for brass. Which were fixed at both ends and pass into the graphite electrodes 5A constant current, change the position of the graphite electrode phase and the contact between the probe,the use of desktop multimeter measured the voltage drop between the two probes by the equation (1) is calculated the resistivity of graphite electrodes.On access to data processing and analysis, and for distribution,As shown in Figure 3, Figure 4: 


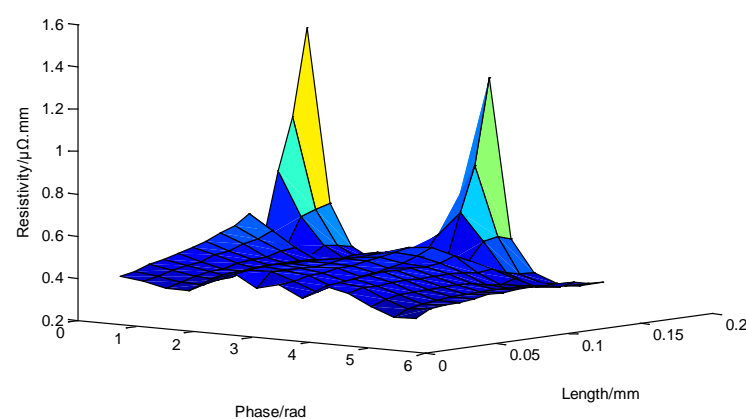

Figure 3 Resistivity distribution of rectangular electrode plate

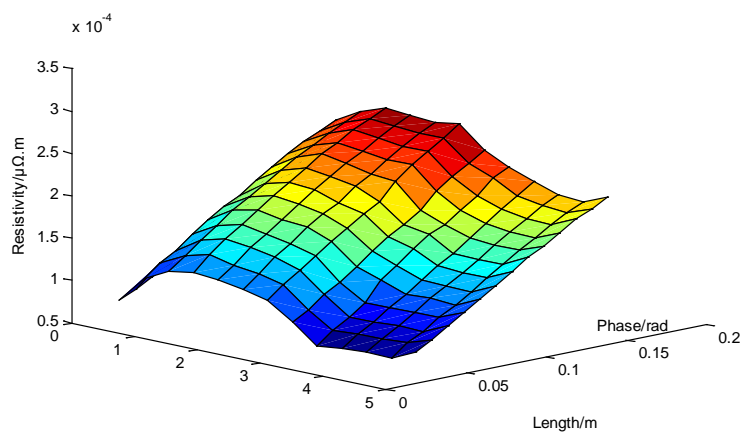

Figure 4 Resistivity distribution of circular electrode

Through the above, we can found in using rectangular disk electrode measurement of its impact on graphite electrode resistivity compared with circular disk electrode larger, and therefore in order to better reflect the graphite electrode resistivity we choose rectangular disk electrode.

In order to further study the effect of phase range on the resistivity of graphite electrode, we resistivity according to the formula, and calculated the resistivity of 16 phase ranges from 2240 sets of data. To analyze the data, get the relationship between the resistivity and phase as shown in Fig 5:

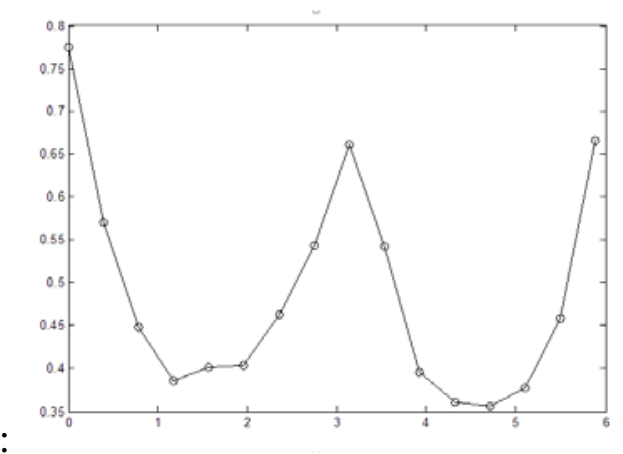

Figure 5 Distribution of resistivity

Through the image, we can infer the relationship between the resistivity and the phase of the triangle, Using the method of curve fitting, least squares fitting, the experimental data are processed and analyzed.

Graphite electrode phase is set $\phi$, Graphite electrode resistivity of function $f(\phi) \in C_{2 \pi}$. Because of the distribution of the electric field density, the electrical resistivity of the cylindrical graphite electrodes can be changed, so the function of the phase and resistivity can be considered as a continuous function,

$$
f(\phi)=a_{1} r_{1}(\phi)+a_{2} r_{2}(\phi)+a_{3} r_{3}(\phi)+\ldots+a_{m} r_{m}(\phi),
$$

Where $r_{k}(\varphi)$ is called the basis function for the sine function $\alpha_{k}$ is undetermined coeffi-cient ( $k=1,2,3, \ldots, m$ ), The number of undetermined coefficients is less than the capacity of the 
experimental data 16, Fitting the standard is to make $\mathrm{n}$ a point $\left(x_{i}, y_{i}\right), i=1,2,3, \ldots, n$ With the square of $y=f\left(\phi_{i}\right) y=f\left(\phi_{i}\right) y=f\left(\phi_{i}\right)$ distance and the smallest. So we can get the following empirical formula by using the least square method:

$$
f(\phi)=1.655 \sin (0.1943 \phi+0.635)+1.197 \sin (0.329 \phi+3.404)+0.167 \sin (1.709 \phi+2.585)
$$

Correctness of the verification formula (3):The relationship between resistivity and phase by phase value, And compare the experimental data with the data of the public figures, as shown in Figure 6.From the graph, we can see that the difference between the actual value and the theoretical value is $0.001 \Omega / \mathrm{mm}$, So the error is within the allowable range, The formula (3) is established.

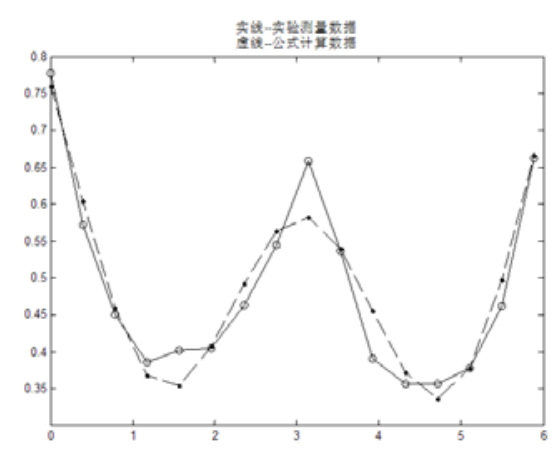

Figure 6 Comparison between theoretical and experimental values

Through the above experimental me can be drawn: in actual industrial measurements can use rectangular disk electrode on the production line of graphite electrode resistivity detection, so the resistivity of the graphite electrode is more accurate.At the same time, the resistivity of the graphite electrode of the rectangular electrode plate is a triangle curve under different phase. And the relationship between the phase and the resistivity is basically in accordance with the formula (3).

\section{Summary}

The resistivity of the graphite is related to not only the length, shape and the graphite itself, but also and working environment of temperature, pressure, humidity, and applied in relation to the graphite at both ends of the current, voltage, pressure, electrode shape and position of the probe and a variety of factors. This experiment using DC probe pressure drop method measuring resistivity of graphite electrode, and analyzes the effect of the resistivity of the graphite electrode with different electrode plate and the different phase of graphite electrode resistivity distribution. The basic requirements of the experimental conditions are in accordance with the basic requirements of the method for determining the electrical resistivity of carbon materials GB/T24525-2009.

\section{References}

[1] Li Shenghua. [M]. Beijing: Metallurgical Industry Press, 1997.1

[2] Cao Junhu. The resistivity of ultra high power consumption for the joint effect of steel [J]. Lanzhou: carbon technology and equipment, 2007.12

[3] Sun Xianshu, Chen Yuzhen. [J]. method of measurement of resistivity of carbon materials: carbon technology, Jilin 1986

[4] Liu Zhangqi. A discussion on the determination of resistivity of carbon products [J]. Jilin: carbon technology, 1994

[5] is the alumni. Graphite electrode material resistivity measurement [J]. Tianshui: practical measurement technology, 1995

[6] Zhang Xinnian. Measuring the resistivity of graphite electrode [J]. Jilin: carbon technology, 1996 
[7] Zhang Liyou. Design and implementation of real time measurement system for graphite electrode [D]. Hunan: Hunan Industrial Science, 2010.6

[8] Ze Yin Wang, Li Zongwei, Jiang Yilong, Wang Yue. The mechanical design of high power carbon electrode resistivity measuring instrument [J]. Heilongjiang: Mechanical Engineer, 2014

[9] Zhang Cong, Cao Haifang, ye Delin. Intelligent test of [J]. graphite electrode resistivity high current carbon technology, Jilin: 2000.3

[10] Ye Delin. Resistivity of carbon products measurement technology and error analysis [J]. Jilin: carbon technology, 1994

[11] Xiao Shutie. Experimental mathematics [M]. University Mathematics in Beijing: Higher Education Press, 2006.5

Communication author: Communication Author: Zhiyi Zhang, male, born in 1966, Professor, Master Supervisor, research direction: research direction for the automation technology theoryand application, motion control theory and application,E-mail:zyzhang6@163.com.

\section{Design of the Chip Kick Mechanics}

At present the main means is low click type and the lever type, low click type is on the bottom of the ball through attack the ball flew over obstacles, this method is able to pick the ball's advantages and makes the energy loss in institutions least, the shortcoming is the ball high requirement of the shape of the electromagnetic valve [11]. Therefore, the development of a high-performance control system of soccer robot has become an urgent desire for soccer robot fans. 